\title{
Teaching NeuroImages: Colchicine-induced vacuolar myopathy
}

Sri Raghav Sista, MD, Maxwell Q. Nyce, MD, Sarah E. Bach, MD, and Gregory M. Blume, MD

Neurolog ${ }^{\circledR}$ 2019;93:e2306-e2307. doi:10.1212/WNL.0000000000008636

Figure Right deltoid muscle biopsy

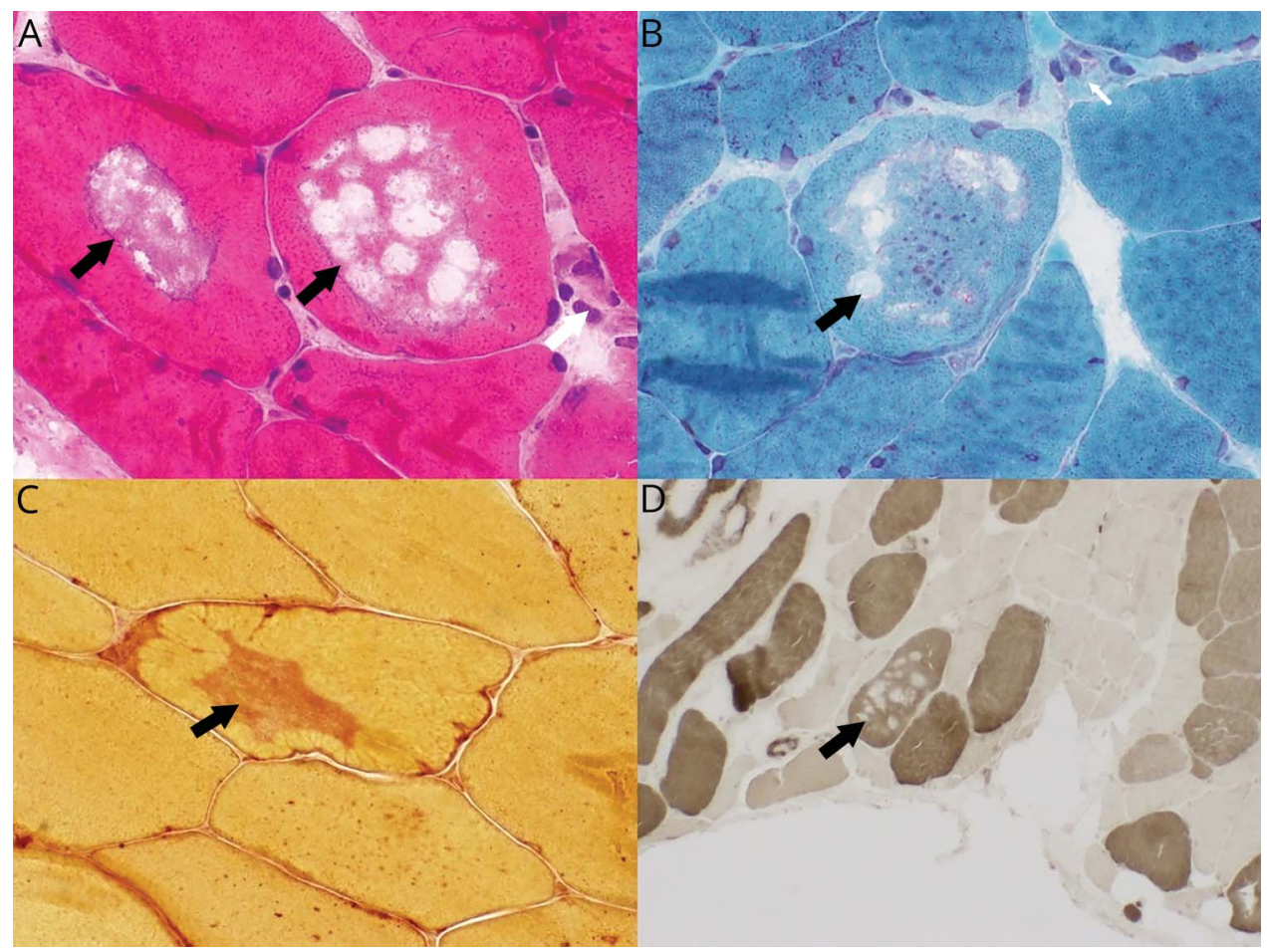

Hematoxylin \& eosin $(A ; 400 x)$ and Gomori trichrome $(B ; 400 x)$ sections show scattered vacuolated and occasional necrotic fibers. Esterase shows increased staining associated with the vacuoles and inclusions (C; 400×). ATPase at pH 4.3 demonstrates vacuoles restricted to type 1 fibers ( $D ; 100 \times)$. Black arrows = vacuoles; white arrows = necrotic fibers.

An 86-year-old man presented with 1 year of insidiously progressive weakness that started around 2 weeks after colchicine initiation for gout. Examination noted proximal more than distal weakness with diffuse hyporeflexia. Creatine kinase was $3,172 \mathrm{U} / \mathrm{mL}$ and electrodiagnostic study was consistent with a myopathic process. Muscle biopsy (figure) showed necrotizing myopathy with vacuoles, as well as mild chronic denervation/reinnervation. Weakness improved after cessation of colchicine.

Colchicine disrupts the microtubules leading to defective movement of lysosomes and formation of autophagic vacuoles. ${ }^{1}$ Autophagic vacuoles can also be seen with chloroquine or hydroxychloroquine therapy, acid maltase deficiency, and various other myopathies. ${ }^{2}$

\section{Correspondence}

Dr. Sista

sri.s.raghavsista@ osfhealthcare.org

\section{MORE ONLINE}

\section{$\rightarrow$ Teaching slides}

links.lww.com/WNL/

B10 


\section{Study funding}

No targeted funding reported.

\section{Disclosure}

The authors report no disclosures relevant to the manuscript. Go to Neurology.org/N for full disclosures.

\begin{tabular}{llll}
\hline Appendix & Authors & & \\
\hline Name & Location & Role & Contributions \\
\hline $\begin{array}{l}\text { Sri Raghav } \\
\text { Sista, MD }\end{array}$ & Peoria & Author & $\begin{array}{l}\text { Study concept or design, analysis or } \\
\text { interpretation of data, drafting/ } \\
\text { revising the manuscript for content, } \\
\text { including medical writing for } \\
\text { content }\end{array}$ \\
\hline $\begin{array}{l}\text { Maxwell } \\
\text { Q. Nyce, }\end{array}$ & Peoria & Author & $\begin{array}{l}\text { Analysis or interpretation of data, } \\
\text { revising the manuscript }\end{array}$ \\
\hline
\end{tabular}

Appendix (continued)

\begin{tabular}{llll}
\hline Name & Location & Role & Contributions \\
\hline $\begin{array}{l}\text { Sarah E. } \\
\text { Bach, MD }\end{array}$ & Peoria & Author & $\begin{array}{l}\text { Analysis or interpretation of data, } \\
\text { drafting/revising the manuscript for } \\
\text { content, including medical writing } \\
\text { for content }\end{array}$ \\
\hline $\begin{array}{l}\text { Gregory } \\
\text { M. Blume, } \\
\text { MD }\end{array}$ & Peoria & Author & $\begin{array}{l}\text { Analysis or interpretation of data, } \\
\text { drafting/revising the manuscript for } \\
\text { content, including medical writing } \\
\text { for content }\end{array}$ \\
\hline
\end{tabular}

\section{References}

1. Katirji B, Kaminski HJ, Ruff RL. Neuromuscular Disorders in Clinical Practice. Berlin: Springer Science \& Business Media, 2013.

2. Nishino I. Autophagic vacuolar myopathy. Semin Pediatr Neurol 2006;13:90-95. 


\section{Neurology}

\section{Teaching NeuroImages: Colchicine-induced vacuolar myopathy \\ Sri Raghav Sista, Maxwell Q. Nyce, Sarah E. Bach, et al. \\ Neurology 2019;93;e2306-e2307 \\ DOI 10.1212/WNL.0000000000008636}

This information is current as of December 9, 2019

\section{Updated Information \&} Services

\section{References}

Subspecialty Collections

Permissions \& Licensing

Reprints including high resolution figures, can be found at: http://n.neurology.org/content/93/24/e2306.full

This article cites 1 articles, 0 of which you can access for free at: http://n.neurology.org/content/93/24/e2306.full\#ref-list-1

This article, along with others on similar topics, appears in the following collection(s):

All Neuromuscular Disease

http://n.neurology.org/cgi/collection/all_neuromuscular_disease Clinical neurology history

http://n.neurology.org/cgi/collection/clinical_neurology_history

EMG

http://n.neurology.org/cgi/collection/emg

Muscle disease

http://n.neurology.org/cgi/collection/muscle_disease

Other toxicology

http://n.neurology.org/cgi/collection/other_toxicology

Information about reproducing this article in parts (figures,tables) or in its entirety can be found online at:

http://www.neurology.org/about/about_the_journal\#permissions

Information about ordering reprints can be found online:

http://n.neurology.org/subscribers/advertise

Neurology $®$ is the official journal of the American Academy of Neurology. Published continuously since 1951, it is now a weekly with 48 issues per year. Copyright (O 2019 American Academy of Neurology. All rights reserved. Print ISSN: 0028-3878. Online ISSN: 1526-632X.

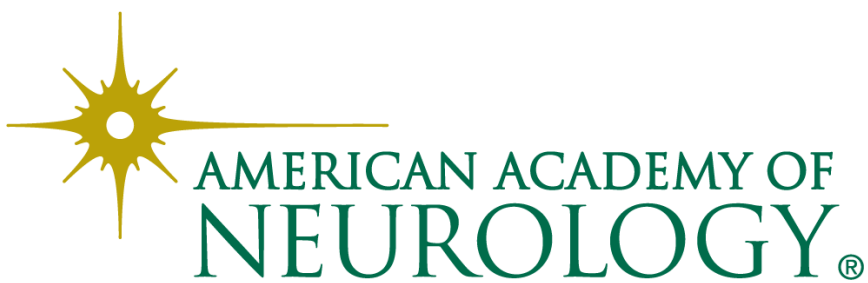

